\title{
Tumor Suppressor miR-637 Is Associated with Cellular Migration, Invasion, and Glioma Diagnosis
}

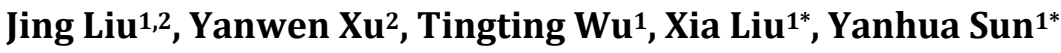 \\ ${ }^{1}$ Department of Pathology, Shenzhen University 1st Affiliated Hospital, Shenzhen Second People's Hospital, School of Medicine, \\ Shenzhen University, Shenzhen, China \\ ${ }^{2}$ Department of Neurosurgery and Shenzhen Key Laboratory of Neurosurgery, Shenzhen University 1st Affiliated Hospital, \\ Shenzhen Second People's Hospital, School of Medicine, Shenzhen University, Shenzhen, China \\ Email: *mdcg2014bl@163.com, *blksyh@126.com
}

How to cite this paper: Liu, J., Xu, Y.W., Wu, T.T., Liu, X. and Sun, Y.H. (2020) Tumor Suppressor miR-637 Is Associated with Cellular Migration, Invasion, and Glioma Diagnosis. International Journal of Clinical Medicine, 11, 516-525. https://doi.org/10.4236/ijcm.2020.119044

Received: August 7, 2020

Accepted: September 4, 2020

Published: September 7, 2020

Copyright $\odot 2020$ by author(s) and Scientific Research Publishing Inc. This work is licensed under the Creative Commons Attribution International License (CC BY 4.0).

http://creativecommons.org/licenses/by/4.0/

\begin{abstract}
Objective: Abnormal miRNA expression is observed in several human tumors; moreover, normal cell regulation can be disrupted by tumor-suppressive or oncogenic miRNAs. We aimed to investigate the role of miR-637 in gliomas. Methods: We assessed miR-637 expression in 98 and 16 gliomas and non-tumoral brain tissues, respectively, using in situ hybridization. We calculated receiver operating characteristic curves to determine the specificity and sensitivity of miR-637 biomarkers. Next, the effects of miR-637 on glioma cell migration and invasion were determined by using the transwell assay. Candidate target genes were identified through Kyoto Encyclopedia of Genes and Genomes pathway enrichment analysis. Results: There was significant miR-637 downregulation in glioma tissues $(P<0.001)$. Further, it showed potential as a diagnostic biomarker for gliomas. In addition, miR-637 suppressed glioma cell migration and invasion. Conclusions: Our findings suggest that miR-637 inhibits glioma invasion and migration and could be a potential diagnostic marker for gliomas. Future studies should examine the potential mechanisms underlying miR-637 as a diagnostic marker and therapeutic target for gliomas.
\end{abstract}

\section{Keywords}

miR-637, Glioma, Diagnosis, Biomarkers

\section{Introduction}

Gliomas are the most common primary brain tumors. World Health Organiza- 
tion (WHO) grade IV glioma, which includes glioblastoma multiform (GBM), is a highly invasive and fatal malignant tumor [1]. GBM can extensively infiltrate normal brain tissue, making surgical resection almost impossible, and postoperative recurrence is common [2]. Therefore, there is a need to elucidate the invasion mechanisms to improve glioma prognosis.

miRNA is a non-coded RNA with a length of 19 - 25 bases. It can directly bind to the 3'-untranslated region of the target mRNA through its seed sequence and inhibit the expression of the encoded protein at the post-transcriptional level [3]. We previously reported that miRNA can regulate cell behavior, including migration, invasion, proliferation, and apoptosis [4] [5]. Based on the role of miRNA in tumor development, it can be classified as tumor-suppressive miRNA (TSmiRNA) or oncogenic miRNA [6]. Several studies have reported that miRNA dysfunction could cause tumorigenesis [7]. However, the specific role of some miRNAs in cancer remains unclear.

miR-637 is among the primate-specific miRNAs first reported by Cummins et al. [8] when using sequence analysis of human colorectal cells. To our knowledge, there have been no studies on miR-637 as an oncogenic miRNA or TS-miRNA. Further, only one study has reported that miR-637 can inhibit tumorigenesis through disruption of signal transducers and activation of transcription 3 signalings in hepatocellular carcinoma [9]. Consequently, we aimed to assess miR-637 expression in gliomas to determine the effects of miR-637 on glioma cell migration and invasion, as well as to explore the possible signaling pathways involved.

\section{Materials and Methods}

\subsection{Tissue Samples and Slide Preparation}

We obtained surgical specimens of 98 gliomas and 16 nontumoral brain tissues (control) from the Pathology Department of the Shenzhen Second People's Hospital, after obtaining written informed consent from the patients. The formalin-fixed, paraffin-embedded (FFPE) tissue sections with a $5-\mu \mathrm{m}$ thickness were cut for subsequent miR-637 in situ hybridization (ISH). This study complied with the principles of the Helsinki Declaration and was approved by the Ethics Committee of the Shenzhen Second People's Hospital.

\subsection{In Situ Hybridization}

FFPE tissue sections, adhered to glass slides, were deparaffinized in xylene and rehydrated using gradient ethanol. We have previously described the details of this procedure [4]. The hybridization images were acquired under a fluorescent microscope.

\subsection{Cell Lines and Cultures}

Human glioma cell lines, U373 and U251 cells, were purchased from the China Academia Sinica Cell Repository (Shanghai, China) and cultured in Dulbecco's 
Modified Eagle Medium (Gibco) that contained 10\% fetal bovine serum (Gibco). All cell lines were incubated at $37^{\circ} \mathrm{C}$ in a humidified incubator with $5 \% \mathrm{CO}_{2} / 95 \%$ air.

\section{4. miR-637 Mimics Transfection}

We purchased the dsRNA oligonucleotides of miR-637 mimics and the scrambled sequence (Scr) of Ribobio. U373 and U251 cells in the miR-637 group and the corresponding Scr group were transfected with the corresponding dsRNA oligonucleotides using X-tremeGENE siRNA Transfection Reagent (Roche).

\subsection{Quantitative Reverse Transcription Polymerase Chain Reaction}

The total RNA from each group was extracted using TRIzol reagent (Invitrogen). miR-637 was quantified using the Bulge-loop miRNA quantitative reverse transcription polymerase chain reaction (qRT-PCR) Detection Kit (Ribobio). U6 was used as the internal miR-637 control. All reactions were performed on a QuantStudio $^{\mathrm{TM}}$ Dx Real-Time PCR Instrument (Thermo Fisher). The fold changes of miR-637 were calculated via the $2^{-\Delta \Delta C t}$ method.

\subsection{Transwell-Chamber Migration and Invasion Assay}

We used transwell inserts (24-well, 8- $\mu \mathrm{m}$ pore size; Millipore) with or without a 1:4 diluted Matrigel (BD Bioscience) coating layer to assess the invasive and migratory abilities of the cells. The cells that reached the lower surface were fixed with methanol and stained with $0.1 \%$ crystal violet. We have previously described the details of this procedure [5]. Lastly, cells from each chamber were counted under a microscope $(\times 400)$.

\subsection{Predication of Candidate Target Genes}

To identify significant candidate target genes of miR-637, we employed four different types of online tools (TargetScan, http://www.targetscan.org/mamm_31/; miRDB, http://www.mirdb.org/; miRTarBase, http://mirtarbase.mbc.nctu.edu.tw/php/index.php; miRWalk, http://zmf.umm.uni-heidelberg.de/apps/zmf/mirwalk2/). Next, we searched for candidate target genes that were identified by at least three tools using the VennDiagram R package.

\subsection{Kyoto Encyclopedia of Genes and Genomes Pathway Enrichment Analysis of Candidate Target Genes}

The Kyoto Encyclopedia of Genes and Genomes (KEGG) (https://www.kegg.jp/) is a database that allows the systematic analysis of gene functions and the linking of genomic information with higher-order functional information. To analyze the candidate target genes at the functional level, we performed KEGG pathway analysis with the Database for Annotation, Visualization, and Integrated Discovery software (DAVID version 6.7; http://www.david.niaid.nih.gov) online 
tool. Significant enrichment was set at $\mathrm{P}<0.05$.

\subsection{Statistical Analysis}

All statistical analyses were performed using SPSS 24.0 software. Results are presented as the mean \pm standard deviation. The normality of distribution was estimated using the Kolmogorov-Smirnov test. Between-group differences were analyzed using a one-way analysis of variance or t-test. To determine the optimal cutoff level of miR-637 expression for glioma diagnosis, we performed a receiver operating characteristic (ROC) curve analysis. Youden's index ( $J=$ sensitivity + specificity - 1) was used to determine the optimal cutoff level [10]. Specifically, the cutoff value for obtaining the highest Youden's index was considered the optimal cutoff level. The level of statistical significance was set at $\mathrm{P}<0.05$. All cell line experiments were performed at least thrice with triplicate samples.

\section{Results}

\section{1. miR-637 Is Down Regulated in Glioma Tissues and Could Be Used for Glioma Diagnosis}

To determine miR-637 expression in gliomas and its association with the WHO grades of gliomas, we used ISH to determine endogenous miR-637 levels in the FFPE specimens. Glioma tissue had significantly lower miR-637 expression than did control tissue $(\mathrm{P}<0.001)$. Further, the expression level was lower when the glioma grade was higher, with the expression in glioblastoma being the lowest $(\mathrm{P}$ $<0.001$; Figure 1). ROC curve analysis of miR-637 expression in both tissue groups revealed an area under the ROC curve of 0.9254 (95\% confidence interval, 0.8698 - 0.9818; $\mathrm{P}<0.0001$; Figure 2(A) and Figure 2(B)). This demonstrated that miR-637 expression in glioma tissues could differentiate between gliomas and nontumoral brain tissues. The optimal cutoff value for glioma diagnosis using the miR-637 labeling index was $\leq 6.015 \%$ (sensitivity, $77.55 \%$; specificity,
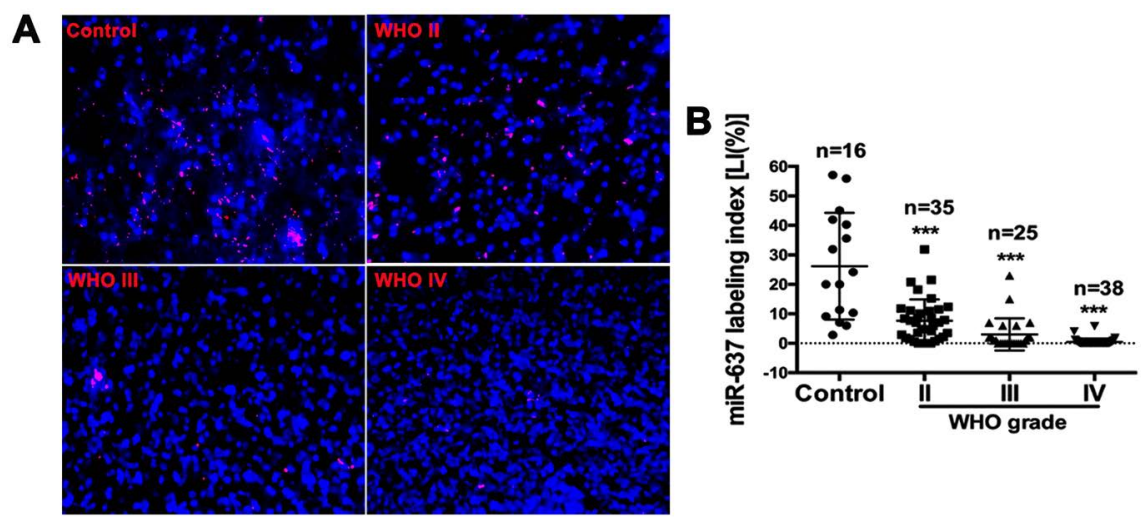

Figure 1. miR-637 downregulation in gliomas. (A) Representative images of the miR-637 in situ hybridization assay. Scale bar, $50 \mu \mathrm{m}$; magnification times $10 \times 20$. (B) Between-group comparisons of miR-637 expression levels (labeling index, LI [\%]) in the formalin-fixed, paraffin-embedded samples. The data are presented as the mean \pm standard deviation. ${ }^{\star * *} \mathrm{P}<0.001$. 

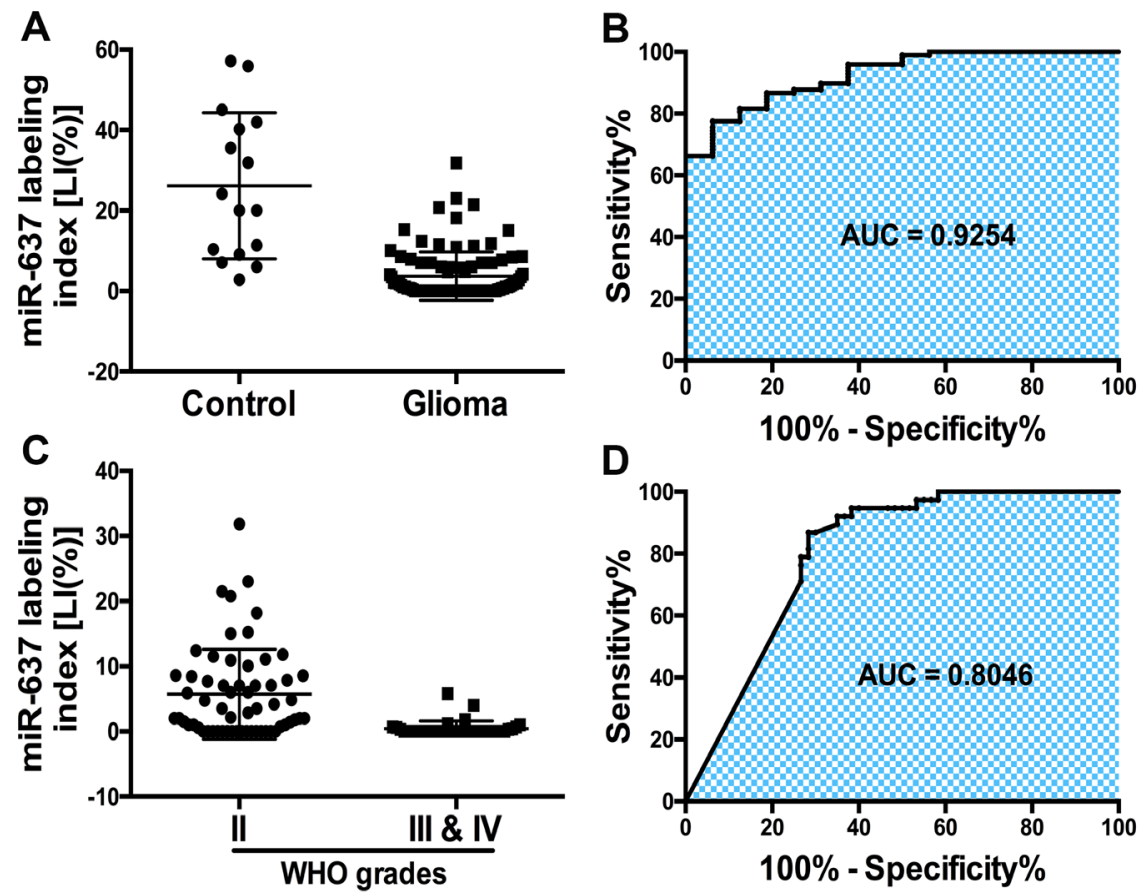

Figure 2. Receiver operating characteristic (ROC) curve analysis to determine the specificity and sensitivity of miR-637 for glioma diagnosis. (A) miR-637 expression in glioma and nontumor brain tissues (control). (B) ROC curve analysis to determine the specificity and sensitivity of miR-637 for glioma diagnosis. The area under the ROC curve is 0.9254 (95\% confidence interval, $0.8698-0.9818$; $\mathrm{P}<0.0001$ ). The optimal cutoff value for glioma diagnosis using the miR-637 labeling index is $\leq 6.015 \%$ (sensitivity, $77.55 \%$; specificity, 93.75\%). (C) miR-637 expression in low-grade gliomas (World Health Organization [WHO] grade II) and high-grade gliomas (WHO III and WHO IV). (D) ROC curve analysis to determine the specificity and sensitivity of miR-637 for the diagnosis of low-grade and high-grade gliomas. The area under the ROC curve is 0.8046 (95\% confidence interval, $0.7190-0.8902 ; \mathrm{P}<0.0001$ ). The optimal cutoff value for glioma diagnosis using the miR-637 labeling index is $\leq 0.7050 \%$ (sensitivity, $86.84 \%$; specificity, $71.67 \%$ ).

93.75\%). ROC curve analysis of miR-637 expression for differentiating between low-grade and high-grade gliomas showed that the area under the ROC curve was 0.8046 (95\% confidence interval, $0.7190-0.8902$; $\mathrm{P}<0.0001$; Figure 2(C) and Figure 2(D)). This indicates that miR-637 expression in glioma tissues could differentiate between glioma grades. The optimal cutoff value for glioma diagnosis using the miR-637 labeling index was $\leq 0.7050 \%$ (sensitivity, $86.84 \%$; specificity, $71.67 \%)$.

\subsection{Validation of Cell Transfection Efficiency}

The transfection efficiency of miR-637 mimics was detected through qRT-PCR. We observed a significant increase in miR-637 levels in U373 and U251 cells after the transfection of miR-637 mimics $(P<0.001$; Figure $3(E))$.

\section{3. miR-637 Inhibits Glioma Cell Migration and Invasion}

We used the transwell-chamber assay to assess the effects of miR-637 on glioma 

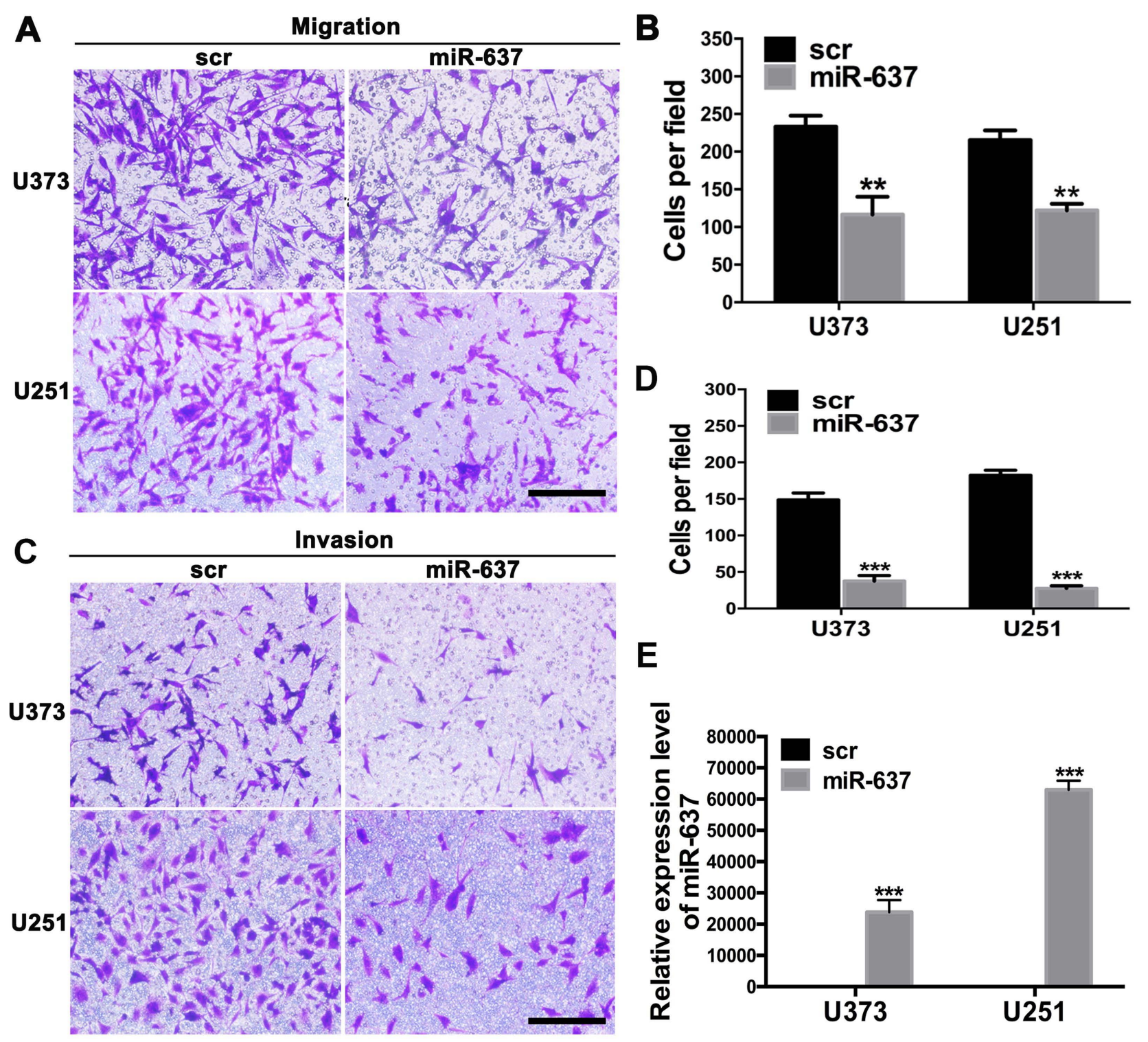

E

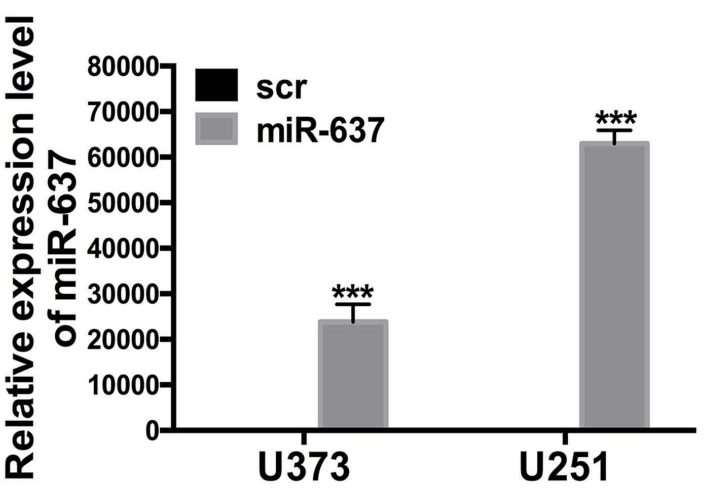

Figure 3. miR-637 inhibits the migration and invasion of glioma cell lines. (A) Transwell-chamber migration assay. Migratory capabilities are reflected by the number of cells per microscopic field that have migrated to the underside of the membrane. Scale bar, $50 \mu \mathrm{m}$. (B) Quantification of the transmembrane migration abilities of cells. All experiments were performed in triplicate, and the results shown in $\mathrm{B}$ are presented as the mean \pm standard deviation (SD). ${ }^{\star *} \mathrm{P}<0.01$. (C) Transwell-chamber invasion assay. The invasive capabilities are reflected by the number of cells per microscopic field that have migrated to the underside of the membrane. Scale bar, $50 \mu \mathrm{m}$. (D) Quantification of the transmembrane invasion abilities of cells. All experiments were performed at least in triplicate, and the results shown in $\mathrm{D}$ are presented as the mean $\pm \mathrm{SD}$. ${ }^{* *} \mathrm{P}<0.001$. (E) Quantitative reverse transcription polymerase chain reaction (qRT-PCR) analysis of miR-637 expression in the cells. The miR-637/U6 ratios in cells transfected with the scrambled sequence are arbitrarily set to 1.0. The results are presented as the mean $\pm \mathrm{SD}$. ${ }^{\star *} \mathrm{P}<$ 0.001 .

cell migration and invasion. Compared with the Scr group, there was a sharp reduction in the number of migrating cells in the miR-637 mimics transfected group $(\mathrm{P}<0.01$; Figure $3(\mathrm{~A})$ and Figure $3(\mathrm{~B})$ ). The transwell-chamber invasion assay showed that miR-637 could effectively inhibit U373 and U251 cell invasion $(P<0.001$; Figure $3(C)$ and Figure 3(D)). Our findings indicate that miR-637 
downregulation could promote U373 and U251 cell migration and invasion.

\subsection{Candidate Target Genes of miR-637 and Pathway Enrichment Analysis}

As shown in Table 1, 136 common candidate target genes were identified from all four tools (Figure 4). The candidate target genes were enriched in KEGG pathways including pathways in cancer and the Rap1 signaling pathway, focal adhesion pathway, and cell adhesion pathway (Table 1). These pathways are involved in cell proliferation, migration, and invasion, which are closely associated with tumor progression and metastasis.

Table 1. Top 10 pathways of candidate target genes identified in the KEGG pathway analysis.

\begin{tabular}{|c|c|c|}
\hline Term & Candidate target genes & $\mathrm{P}$-value \\
\hline Pathways in cancer & COLA1, SMA3, GNAI, PRKCA, DVL3 & 0.000472842 \\
\hline Leukocyte transendothelial migration & GNAI, VAV2, CLDN2, THY1, PRKCA & $6.46 \mathrm{E}-05$ \\
\hline Rap1 signaling pathway & $G N A D, V A V 2, A D C Y 1, P R K C A$ & 0.006265224 \\
\hline Proteoglycans in cancer & TFAP4, VAV2, HPSE2, PRKCA & 0.005677188 \\
\hline Focal adhesion & $V A V 2, I T G A 10, C O L A A 1, P R K C A$ & 0.005489688 \\
\hline Purine metabolism & $A K 3, A D C Y 1, P K M, P R I M 2$ & 0.003350668 \\
\hline Oxytocin signaling pathway & NFATCA, GNAI, ADCY1, PRKCA & 0.002294611 \\
\hline Cell adhesion molecules & $N F A S C, C D 40 L G, C L D N 2, S D C 3$ & 0.001734685 \\
\hline Wnt signaling pathway & NFATCA, SMAD3, DVL3, PRKCA & 0.001611186 \\
\hline Chagas disease (American trypanosomiasis) & $S M A D 3, C A L R, C E R S 1, G N A D$ & 0.000511789 \\
\hline
\end{tabular}

KEGG, Kyoto Encyclopedia of Genes and Genomes.

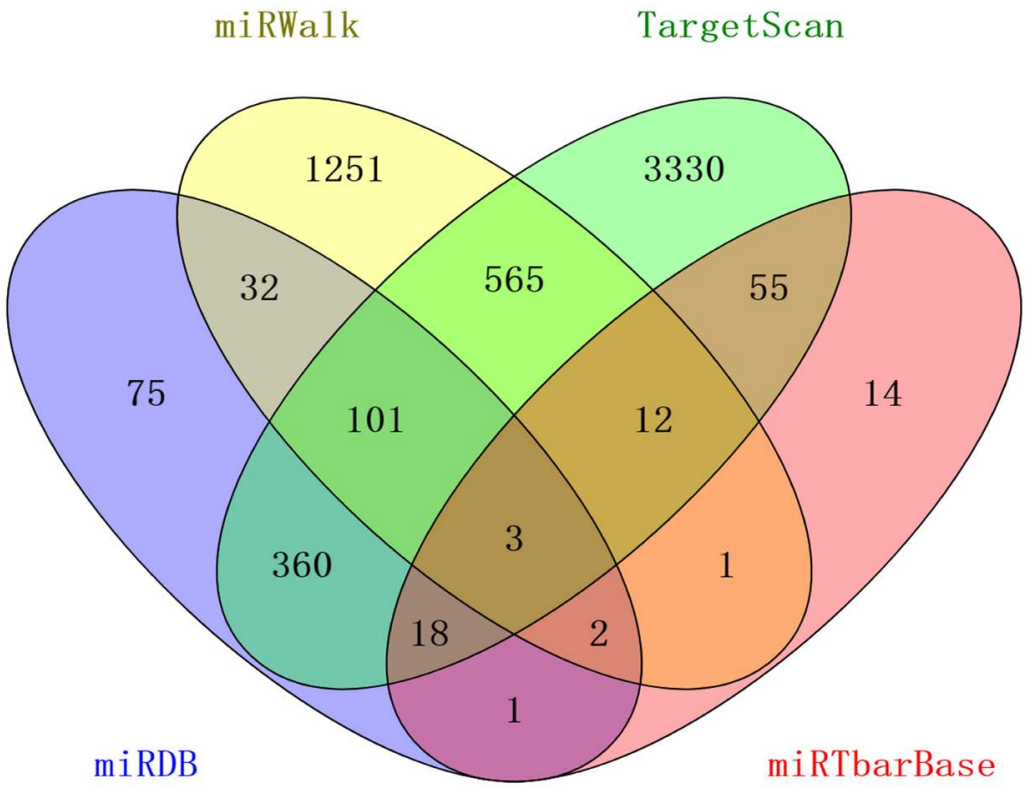

Figure 4. Venn diagram of miR-637 target-gene prediction. 


\section{Discussion}

Only a few studies have investigated the functions of miR-637, which is a primate-specific miRNA first reported in 2006 by Cummins et al. [8]. For example, Dai et al. [11] reported miR-637 down-regulation in the context of primary immunoglobulin A nephropathy. Riccardo et al. [12] reported that the ATP6V0A1 polymorphism and miR-637 could cause essential hypertension. Zhang et al. [13] reported that miR-637 maintains the balance between adipocytes and osteoblasts by directly targeting Osterix. A limited number of cancer-related studies have described the role of miR-637 in different tumors, possibly as a TS-miRNA or oncogenic miRNA. Regarding gliomas, only two studies have reported miR-637 as a TS-miRNA [14] [15].

Tumorigenesis is a complex process that is related to the activation of various oncogenes or dysfunction of tumor suppressor genes. We aimed to elucidate the role of miR-637 in gliomas. Using ISH, we assessed miR-637 expression in 98 human glioma tissues and 16 nontumoral brain tissues. Further, we evaluated the effects of miR-637 mimics on glioma cell lines using in vitro experiments. We conducted transwell-chamber migration and invasion assays to assess the effects of miR-637 on cell migration and invasion. Compared with nontumoral brain tissues, miR-637 expression was relatively low in glioma tissues and was negatively related to the WHO grade. Additionally, the transfection of miR-637 mimics significantly inhibited the migration and invasion of U373 and U251 cells, which further confirmed the inhibitory effects of miR-637 on gliomas. We conducted bioinformatics to analyze the target genes of miR-637 enriched in KEGG pathways and found that they were closely associated with tumor cell invasion and migration. Future studies should elucidate the potential molecular mechanisms and specific target genes of miR-637 in gliomas.

There were 136 common candidate target genes predicted as putative target genes from all four tools. KEGG pathway analysis revealed that pathways in cancer, leukocyte transendothelial migration, Rap1 signaling, proteoglycans in cancer, focal adhesion, purine metabolism, oxytocin signaling, cell adhesion molecules, Wnt signaling, and Chagas disease (American trypanosomiasis) were the top 10 enriched pathways of the predicted target genes. These pathways play vital roles in the regulation of cell migration, invasion, and proliferation. There were other potential target genes associated with tumorigenesis, including $E C M$, PTGIS, SRSF1, and TRPM7. Further studies are needed to explore the candidate targets of miR-637 and verify the mechanisms underlying its inhibitory effects on migration and invasion.

\section{Conclusion}

Our findings indicated that miR-637 downregulation occurred to a greater extent in glioma tissues than in nontumoral brain tissues. Transfection of miR-637 mimics inhibited migration and invasion of U373 and U251 cells. These results suggest that miR-637 could be a tumor suppressor in gliomas. Moreover, our 
findings indicate that miR-637 is not only a potential diagnostic biomarker but also a potential therapeutic target for gliomas. Future studies should verify the target genes of miR-637 and identify the potential regulatory pathways.

\section{Acknowledgements}

This research did not receive any specific grant from funding agencies in the public, commercial, or not-for-profit sectors.

\section{Conflicts of Interest}

The authors declare no conflicts of interest regarding the publication of this paper.

\section{References}

[1] Wilson, T.A., Karajannis, M.A. and Harter, D.H. (2014) Glioblastoma Multiforme: State of the Art and Future Therapeutics. Surgery Neurology International, 5, 64. https://doi.org/10.4103/2152-7806.132138

[2] Geiger, G.A., Fu, W. and Kao, G.D. (2008) Temozolomide-Mediated Radiosensitization of Human Glioma Cells in a Zebrafish Embryonic System. Cancer Research, 68, 3396-3404. https://doi.org/10.1158/0008-5472.CAN-07-6396

[3] Liu, M., Tang, Q., Qiu, M., Lang, N., Li, M., Zheng, Y., et al. (2011) miR-21 Targets the Tumor Suppressor RhoB and Regulates Proliferation, Invasion and Apoptosis in Colorectal Cancer Cells. FEBS Letters, 585, 2998-3005. https://doi.org/10.1016/j.febslet.2011.08.014

[4] Liu, J., Xu, J., Li, H., Sun, C., Yu, L., Li, Y., et al. (2015) miR-146b-5p Functions as a Tumor Suppressor by Targeting TRAF6 and Predicts the Prognosis of Human Gliomas. Oncotarget, 6, 29129-29142. https://doi.org/10.18632/oncotarget.4895

[5] Li, H., Yu, L., Liu, J., Bian, X., Shi, C., Sun, C., et al. (2017) miR-320a Functions as a Suppressor for Gliomas by Targeting SND1 and $\beta$-Catenin, and Predicts the Prognosis of Patients. Oncotarget, 8, 19723-19737.

https://doi.org/10.18632/oncotarget.14975

[6] Pernaute, B., Spruce, T., Smith, K.M., Sánchez-Nieto, J.M., Manzanares, M., Cobb, B., et al. (2014) MicroRNAs Control the Apoptotic Threshold in Primed Pluripotent Stem Cells through Regulation of BIM. Genes \& Development, 28, 1873-1878. https://doi.org/10.1101/gad.245621.114

[7] Garzon, R., Calin, G.A. and Croce, C.M. (2009) MicroRNAs in Cancer. Annual Review of Medicine, 60, 167-179. https://doi.org/10.1146/annurev.med.59.053006.104707

[8] Cummins, J.M., He, Y., Leary, R.J., Pagliarini, R., Diaz, L.A., Sjoblom, T., et al. (2006) The Colorectal microRNAome. Proceedings of the National Academy of Sciences of the United States of America, Vol. 103, 3687-3692. https://doi.org/10.1073/pnas.0511155103

[9] Zhang, J.F., He, M.L., Fu, W.M., Wang, H., Chen, L.Z., Zhu, X., et al. (2011) Primate-Specific microRNA-637 Inhibits Tumorigenesis in Hepatocellular Carcinoma by Disrupting Signal Transducer and Activator of Transcription 3 Signaling. Hepatology, 54, 2137-2148. https://doi.org/10.1002/hep.24595

[10] Salvadó, G., Molinuevo, J.L., Brugulat-Serrat, A., Falcon, C., Grau-Rivera, O., Suárez-Calvet, M., et al. (2019) Centiloid Cut-Off Values for Optimal Agreement 
between PET and CSF Core AD Biomarkers. Alzheimer's Research \& Therapy, 11, Article No. 27. https://doi.org/10.1186/s13195-019-0478-Z

[11] Dai, Y., Sui, W., Lan, H., Yan, Q., Huang, H. and Huang, Y. (2008) Microarray Analysis of Micro-Ribonucleic Acid Expression in Primary Immunoglobulin A Nephropathy. Saudi Medical Journal, 29, 1388-1393.

[12] Coutu, R. and Condorelli, G. (2011) ATP6V0A1 Polymorphism and microRNA-637: A Pathogenetic Role for microRNAs in Essential Hypertension at Last? Circulation Cardiovascular Genetics, 4, 337-338. https://doi.org/10.1161/CIRCGENETICS.111.960591

[13] Zhang, J.F., Fu, W.M., He, M.L., Wang, H., Wang, W.M., Yu, S.C., et al. (2011) MiR-637 Maintains the Balance between Adipocytes and Osteoblasts by Directly Targeting Osterix. Molecular Biology of the Cell, 22, 3955-3961. https://doi.org/10.1091/mbc.e11-04-0356

[14] Que, T., Song, Y., Liu, Z., Zheng, S., Long, H., Li, Z., et al. (2015) Decreased miRNA-637 Is an Unfavorable Prognosis Marker and Promotes Glioma Cell Growth, Migration and Invasion via Direct Targeting Akt1. Oncogene, 34, 4952-4963. https://doi.org/10.1038/onc.2014.419

[15] Yi, W., Li, D., Guo, Y., Zhang, Y., Huang, B. and Li, X. (2016) Sevoflurane Inhibits the Migration and Invasion of Glioma Cells by Upregulating microRNA-637. International Journal of Molecular Medicine, 38, 1857-1863. https://doi.org/10.3892/ijmm.2016.2797 